Meta

Journal des traducteurs

Translators' Journal

\title{
Étude comparative et diachronique des concepts ecosystem et écosystème
}

\section{Pascaline Dury}

Volume 44, numéro 3, septembre 1999

URI : https://id.erudit.org/iderudit/002690ar

DOI : https://doi.org/10.7202/002690ar

Aller au sommaire du numéro

\section{Éditeur(s)}

Les Presses de l'Université de Montréal

ISSN

0026-0452 (imprimé)

1492-1421 (numérique)

Découvrir la revue

Citer cet article

Dury, P. (1999). Étude comparative et diachronique des concepts ecosystem et écosystème. Meta, 44(3), 485-499. https://doi.org/10.7202/002690ar

\section{Résumé de l'article}

La terminologie diachronique reste encore un domaine relativement peu exploré par les ouvrages consacrés entièrement à la terminologie. Pourtant, l'angle de réflexion qu'elle procure est à la fois original et riche. Elle apporte en effet un point de vue différent sur les concepts, le point de vue historique, qui peut être très utile aux traducteurs. L'évolution diachronique des concepts ÉCOSYSTÈME et ECOSYSTEM, à la fois en français et en anglais, montre en effet que l'identité conceptuelle ne peut pas être décrétée à priori en terminologie. De la même façon, des concepts qui paraissent "anodins" ou simples d'accès à première vue (c'est le cas des concepts COMMUNITY et COMMUNAUTÉ) peuvent se révéler en fait relativement complexes. Cette rapide étude de la langue de l'écologie au moyen d'un concept clé, montre donc que la terminologie diachronique peut devenir l'outil de travail indispensable du traducteur car elle intervient à différents niveaux de réflexion.
Tous droits réservés @ Les Presses de l'Université de Montréal, 1999
Ce document est protégé par la loi sur le droit d'auteur. L'utilisation des services d'Érudit (y compris la reproduction) est assujettie à sa politique d'utilisation que vous pouvez consulter en ligne.

https://apropos.erudit.org/fr/usagers/politique-dutilisation/ 


\title{
ÉTUDES TERMINOLOGIQUES ET LINGUISTIQUES
}

\section{Étude comparative et diachronique des concepts ecosystem et écosystème}

\author{
pascaline dury \\ Université Lumière Lyon-2, Lyon, France
}

\begin{abstract}
RÉSUMÉ
La terminologie diachronique reste encore un domaine relativement peu exploré par les ouvrages consacrés entièrement à la terminologie. Pourtant, l'angle de réflexion qu'elle procure est à la fois original et riche. Elle apporte en effet un point de vue différent sur les concepts, le point de vue historique, qui peut être très utile aux traducteurs. L'évolution diachronique des concepts ÉCOSYSTĖME et ECOSYSTEM, à la fois en français et en anglais, montre en effet que l'identité conceptuelle ne peut pas être décrétée à priori en terminologie. De la même façon, des concepts qui paraissent «anodins» ou simples d'accès à première vue (c'est le cas des concepts COMMUNITY et COMMUNAUTÉ) peuvent se révéler en fait relativement complexes. Cette rapide étude de la langue de l'écologie au moyen d'un concept clé, montre donc que la terminologie diachronique peut devenir l'outil de travail indispensable du traducteur car elle intervient à différents niveaux de réflexion.
\end{abstract}

\begin{abstract}
Although the history of terms and concepts proves to be very productive in the terminology of some domains, here the domain of ecology, it is still only rarely mentionned in books and articles which deal with terminology. However, despite what one may still consider as a generally unfavourable climate, the history of concepts needs to be developped for at least two reasons. First, it shows that two concepts are not always strictly identical, even if they wear names which appear to be equivalent or translated from one language into another. This is the case for the terms écosystème and ecosystem. Second, concepts that translators may consider as well-known and easy to understand, sometimes appear to be more complex than what they seem to be. This is the case for the concepts COMMUNITY and COMMUNAUTÉ. A quick study of the history of the concepts ECOSYSTEM and ÉCOSYSTĖME, shows that translators and terminologists would higly benefit from a more regular use of the historical information gathered on concepts and terms.
\end{abstract}

\section{INTRODUCTION}

À en juger par le peu de publications consacrées aux analyses de type diachronique, on pourrait croire qu'il s'agit d'une activité marginale de la terminologie. En effet, encore trop peu d'articles et d'ouvrages strictement dédiés à la terminologie se penchent sur l'évolution historique des termes et des concepts. Pourtant, les réflexions de type diachronique sont riches d'enseignement à la fois pour le terminologue et le traducteur, car elles offrent une approche nouvelle des termes et des concepts ${ }^{1}$. Seule 
la terminologie diachronique peut montrer à quel point l'évolution d'un concept peut être complexe et inattendue, et lourde de conséquences pour la dénomination qu'il porte. Il y a très souvent inadéquation entre l'évolution d'une notion et celle de sa dénomination, qui ne varie que très rarement, même si le concept ne cesse de se modifier, de se complexifier. À l'inverse, une analyse diachronique peut montrer que la constitution d'un concept peut s'accompagner d'un véritable foisonnement terminologique, comme c'est le cas pour ÉCOSYSTÈM E² évoqué dans cet article.

Dans l'intérêt du traducteur, il est possible de compléter la richesse de l'analyse diachronique par celle de l'analyse comparative entre deux langues, ici le français et I'anglais. En effet, on sait que le traducteur spécialisé ne doit pas seulement traduire une forme, mais doit aussi s'intéresser à la notion qui l'accompagne. II doit, par conséquent, vivre en bonne intelligence avec la notion et connaître son évolution historique quelle que soit la langue de travail. II doit également s'assurer que le concept de la langue d'arrivée n'est pas seulement homologue, mais bien identique au concept de la langue de départ.

Cet article prend donc la forme d'un véritable plaidoyer pour la terminologie diachronique et comparative. Pour ce faire, nous avons choisi d'illustrer notre propos par la langue de l'écologie, car elle est tout à fait caractéristique et, à l'intérieur de cette science, nous avons choisi d'étudier plus en détail les concepts ÉCOSYSTÈME et ECOSYSTEM .

\section{CHOIX DU DOMAINE D'APPLICATION}

\subsection{Pourquoi parler d'écologie?}

L'écologie est une science digne d'intérêt d'un point de vue terminologique, et ce, pour plusieurs raisons qui lui sont propres. Tout d'abord, c'est une science récente dont l'indépendance scientifique ne date pas de plus d'un siècle ${ }^{3}$, ce qui représente une période de vie plutôt courte si on la compare à d'autres sciences comme la biologie ou la chimie, par exemple, qui existent depuis plusieurs siècles déjà. Par conséquent, l'écologie offre un parcours historique assez caractéristique, marqué notamment par un nombre important d'emprunts conceptuels à des sciences connexes comme la biologie, la zoologie et la botanique, entre autres. Pour cette raison, beaucoup d'auteurs scientifiques ${ }^{4}$ considèrent l'écologie comme une science «composite», issue de la cristallisation d'autres sciences et n'ayant pas, pour ainsi dire, d'identité véritable et de terminologie propre. L'analyse du concept ÉCOSYSTÈME qui suit tend à prouver le contraire.

Cette science est récente, nous l'avons dit, et par conséquent, un certain nombre de ses concepts fondamentaux sont encore en pleine évolution, en pleine maturation, faisant de l'écologie un formidable outil d'observation et de travail pour les terminologues. Ceux-ci deviennent alors les témoins (et pourquoi pas les auteurs) de naissances et de modifications conceptuelles et terminologiques régulières.

Enfin, l'écologie est à la mode, elle est devenue la préoccupation principale de nombreux pays développés mais aussi de nombreux individus, associations et partis politiques à travers le monde. Son objet est donc discuté, encouragé, documenté, transmis, fai sant de l'écologie une science ouverte à une vulgarisation de plus en plus systématique et de plus en plus rapide. Cependant, cet engouement provoque des 
altérations au sein même du domaine, modifiant sa terminologie et la construction notionnelle qui le caractérise. D'ailleurs, aujourd'hui, parle-t-on encore de la même science, lorsque l'on évoque les relations entre les organismes vivants et leur milieu naturel telles que les envisageait Haeckel, par exemple, et lorsque l'on considère l'écologie telle que les partis politiques nous la décrivent, synonyme de surpopulation, de pollution et de catastrophes naturelles? Nous verrons qu'il s'est opéré une véritable césure au sein du concept entre l'écologie classique, telle qu'elle a été définie par Haeckel, et les préoccupations humaines, politiques et sociales qui de plus en plus se regroupent sous les termes écologisme et environnementalisme, car ceci bien sûr ne va pas sans conséquences terminologiques.

\subsection{L'écosystème, un concept caractéristique de la science}

Le concept ÉCOSYSTÈME et le concept équivalent en anglais, ECOSYSTEM (I'équivalence ne présupposant pas pour l'instant l'identité entre les deux concepts), représentent des notions fondamentales de l'écologie. Cependant, ils ont la particularité, au contraire d'autres notions importantes de la science comme BIOSPHÈRE, CLIMAX, et NICHE ${ }^{5}$, pour n'en citer que quelques-unes, de ne pas avoir été empruntés à des sciences connexes mais d'être de facture purement écologique. En d'autre termes, le concept ÉCOSYSTĖME est apparu pour la première fois dans un contexte écologique et n'existe que dans cette science. II est, en cela, caractéristique.

De plus, l'étude diachronique ${ }^{6}$ des concepts ÉCOSYSTÈME et ECOSYSTEM est riche d'enseignements car elle fait apparaître des phénomènes terminologiques particuliers, notamment le foisonnement terminologique qui accompagne parfois la constitution et la maturation d'un concept. On constatera à cette occasion le parallélisme entre mouvements conceptuels et mouvements terminologiques, alors que très souvent les concepts scientifiques évoluent sans que les dénominations qui les désignent en soit modifiées pour autant.

Enfin, une étude comparative entre le français et l'anglais montre qu'il y a des différences terminologiques importantes entre les deux langues en matière d'écosystème, cas plutôt rare en écologie, un domaine particulièrement marqué par les emprunts à la langue anglaise.

\section{LA CONSTITUTION DU CONCEPT}

\subsection{Tansley, inventeur du terme ecosystem}

La paternité du terme ecosystem (construit sur la contraction du syntagme ecological system) revient à l'écologue britannique Tansley (1935: 299), qui observe:

It is the system so formed which, from the point of view of the ecologist, are the basic units of nature on the face of the earth. Our natural human prejudices force us to consider the organisms [...] as the most important parts of these systems, but certainly the inorganic «factors» are also parts. [...] These «ecosystems», as we may call them, are of the most various kinds and size.

Lorsqu'il décrit le terme ecosystem pour la première fois, Tansley insiste sur le fait qu'il prend system dans le sens avec lequel ce dernier est utilisé en physique et il précise que le concept ECOSYSTEM , tel qu'il l'envisage, n'existe pas comme une réali ité pal pable, 
mais bien plutôt comme le produit d'une création mentale qu'il est possible d'isoler en instaurant une frontière entre elle et l'imaginaire. Soyons claire: il s'agit ici d'une réalité abstraite pourtant formée à partir d'éléments qui eux sont concrets. Tansley définit également le concept ECOSYSTEM comme étant un ensemble construit par les relations qu'entretiennent les espèces vivantes et l'habitat physique qui leur permet de se développer. II insiste en outre sur le caractère mouvant de cet ensemble: il dépend de facteurs exogènes ou externes comme la température, l'ensoleillement, I'humidité, etc. et de facteurs internes comme la taille des populations d'êtres vivants qui l'occupent. L'écosystème se modifie donc en fonction de ces facteurs.

Cependant, si Tansley est le premier scientifique chez qui on trouve des traces d'ecosystem, et s'il est même considéré comme le créateur du terme ${ }^{7}$, il semble toutefois indéniable que le concept existait déjà à l'état de germe dans d'autres travaux écologiques, américains notamment. C'est ainsi que le concept COM MUNITY, à I'origine, était considéré comme un concept homologue du concept ECOSYSTEM . Nous rapportons à ce sujet les propos d'Egerton (1977: 357) :

Communities continued as major objects of study for ecologists and, in 1935, were rechristened and incorporated in an extended concept «ecosystems» by Sir Arthur George Tansley, a prominent British plant ecologist.

II faut ajouter à cela que le terme ecosystem apparaît à une époque où l'écologie américaine développe une conception organiciste de la science. En effet, dès 1916, de nombreux auteurs comme Clements défendent l'idée que les végétaux se comportent comme de véritables êtres vivants, qui s'organisent en communautés capables d'évoluer et de se transformer au fil du temps et en fonction des facteurs extérieurs. Cette école de pensée, qui se base sur le caractère évolutif des organismes, conduit à une conception dynamique de l'écologie. Clements émet d'ailleurs l'idée que ce qu'il appelle les communautés biotiques ${ }^{8}$ (biotic communities) ne sont pas fixes et immuables, mais au contraire sujettes aux changements dans le temps. Elles se développent ainsi progressivement jusqu'à atteindre leur seuil maximum d'expansion, le dimax. Clements, à qui revient la paternité du terme climax utilisé dans un sens écologique, considère d'ailleurs que l'ensemble des plantes appartenant à la même communauté biotique forme ce qu'il appelle un superorganisme (superorganism), qui possède fonctions et structures. Cette conception organiciste des communautés biotiques provoquera de vives réactions dans les milieux scientifiques, et c'est dans ce cadrelà que Tansley utilise le terme ecosystem pour la première fois. II affirme en effet que si les communautés végétales se comportent comme des organismes, il ne faut pas pour autant affirmer qu'elles en sont et il préférera utiliser le terme quasi organism pour les dénommer avant d'inventer, quelques années plus tard, celui d'ecosystem? ${ }^{9}$.

Tansley, plus qu'une simple clarification d'ordre terminologique, venait également de donner une nouvelle extension au concept. Désormais, le concept ECOSYSTEM n'est plus considéré comme un simple système relativement homogène, dans lequel un équilibre particulier s'établit dans des réseaux biologiques spécifiques, mais bien plutôt comme un système dans lequel il se crée une véritable interdépendance entre le monde vivant et le support physique. 


\section{2. À propos du concept COMMUNITY}

La mention du concept COMMUNITY nous permet d'ouvrir une parenthèse terminologique qui nous semble importante. Elle concerne les notions qui nous semblent anodines et bien connues, comme c'est le cas pour COM MUNITY, par exemple. M algré leur apparente simplicité, ces dernières peuvent représenter un véritable piège pour les traducteurs qui travaillent dans un contexte bilingue, ici françaisanglais. En effet, on serait tenté, étant donné la simplicité apparente du terme anglais community, de le traduire systématiquement en français par le terme, tout aussi anodin, de communauté. Pourtant, le terme community peut être traduit de nombreuses façons différentes dans la langue française de l'écologie, puisqu'il peut désigner un peuplement, une biocénose et, nous venons de le voir, un écosystème (des termes qui ne sont en aucun cas synonymes). Nous touchons là un point de terminologie essentiel. II est impossible de considérer les termes community et communauté comme des équivalents stricts, car les concepts qu'ils désignent ne sont pas identiques, mais seulement homologues. Nous reprenons ici une théorie émise par Thoiron (1994) selon laquelle, en terminologie, l'identité conceptuelle ne peut pas être décrétée à priori. Il convient de faire la différence entre des concepts homologues (qui ne se correspondent donc que partiellement) et des concepts identiques. II est d'autant plus difficile de s'apercevoir que deux concepts ne sont pas identiques si les termes qui les dénomment sont relativement anodins, en particulier à cause de leurs caractéristiques formelles, et perçus comme des équivalents linguistiques, comme c'est le cas ici.

En effet, une communauté, au sens écologique, désigne un peuplement d'espèces différentes vivant dans un même écosystème. Aguesse (1971: 215) donne la définition suivante du terme communauté:

Ensemble des organismes appartenant aux règnes végétal et animal qui vivent dans un habitat donné. Ce terme implique obligatoirement la présence simultanée de plusieurs espèces différentes.

Le terme community, par contre, ne donne pas l'idée que les organismes qui cohabitent doivent être de nature différente. D'ailleurs, l'ensemble des définitions que nous avons pu lire sur le terme community nous laissent finalement penser que le concept COM M U N ITY ${ }^{10}$ possède une extension plus large que le concept COMMUNAUTÉ. En effet, la notion de COM M UNITY, moins restrictive, comporte bien l'idée d'une limitation du groupe d'organismes dans l'espace mais, en revanche, ne donne aucune information sur la nature de ces organismes, alors que la notion de COM MUNAUTÉ implique que les espèces soient de nature différente. II paraît donc souhaitable, à la lueur de cette analyse, de ne pas traduire systématiquement le terme community par communauté, mais de préférer parfois, selon les cas, les termes peuplement, biocénose et parfois aussi écosystème.

Nous constatons une fois de plus que la terminologie (ici comparative et diachronique) est un outil de travail indispensable pour le traducteur, qui doit non seulement traduire des signes, mais aussi des notions, et qui doit donc apprendre à connaître ces dernières, même si elles lui paraissent anodines, car le cas de COM M UNITY le montre bien, elles peuvent être sources d'ambiguïtés. 


\subsection{Les travaux précurseurs du concept en Europe}

Les concepts ECOSYSTEM et ÉCOSYSTÈME sont particulièrement intéressants à étudier parce qu'ils se sont développés à la fois aux États-Unis, comme nous l'avons vu avec les travaux de Clements, mais aussi en Europe avec Tansley bien sûr et avec bien d'autres avant lui. En effet, ce dernier se place comme l'héritier de toute une série de travaux sur le sujet, le concept ECOSYSTEM se constituant depuis plusieurs décennies, voire depuis plusieurs siècles déjà.

Ainsi, les facteurs extérieurs qui constituent une variable importante du concept font l'objet de travaux dès le début du xix ${ }^{\mathrm{e}}$ siècle. De nombreux savants réfléchissaient sur le rôle capital joué dans la nature par les températures, les préci pitations, la latitude et l'altitude notamment. Les botanistes de l'époque, Humboldt, Candolle et Warning pour ne citer qu'eux, avaient même contribué à mettre au point un système de classement végétal qui ne prenne pas seulement en compte l'aspect physique de la plante, comme c'était le cas jusque-là, mais aussi sa répartition géographique et ses rapports avec le milieu. De ce fait, ils avaient étudié avec précaution tout ce qui touchait à la température, I'humidité du sol et le climat et étaient déjà parvenus à la conclusion que les plantes poussaient non pas au hasard, mais plutôt dans des ensembles géographiques et physiques bien particuliers. Ces travaux donnèrent même naissance à une terminologie bien précise, en français notamment, qui devait être plus tard remplacée par le terme écosystème. Le terme biome et après lui celui de groupement végétal, développé par $C$ andolle au xix ${ }^{\mathrm{e}}$ siècle, sont considérés comme les termes précurseurs d'écosystème. Deléage (1992: 43) note d'ailleurs à ce sujet que la notion de BIOME «aboutit, avec notamment les œuvres d'Alphonse de Candolle et Eugène Warming, à la notion de «groupement végétal », qui créera elle-même les conditions de possibilité du concept d'écosystème».

\section{CONSTITUTION CONCEPTUELLE ET FOISONNEMENT TERMINOLOGIQUE}

\subsection{Adoption et implantation du terme ecosystem}

Nous décrivons ici un phénomène qui est assez rare dans I'histoire de l'écologie pour être souligné. Comme nous l'avons déjà dit, une partie des concepts fondamentaux de la science sont issus d'emprunts directs à d'autres sciences voisines comme la zoologie ou la botanique et, dans la grande majorité de ces cas, l'emprunt notionnel s'accompagne d'un emprunt terminologique, introduisant du même coup dans la science un concept et un terme nouveaux.

Parfois ce double emprunt se fait directement à une langue voisine, dans le même domaine: ainsi, l'écologie française a-t-elle directement emprunté le concept CLIM AX (et donc le terme) à l'écologie américaine. Cette dernière avait développé, nous l'avons vu, une vision dynamique de l'évolution des végétaux, alors que l'Europe et la France, en particulier, favorisaient une étude plus statique de la végétation, essentiellement basée sur leur morphologie. L'adoption du concept CLIM AX (et du terme qui l'accompagne) ne vient donc remplacer aucun concept ou aucun terme existant déjà.

Dans le cas de l'écosystème au contraire, il est intéressant de noter que des travaux ont lieu dans plusieurs pays à la fois, ceux-ci développant des notions homo- 
logues qui reçoivent des dénominations dans la langue locale. Nous pouvons ainsi dresser une liste des vocables anglais qui ont précédé l'adoption du terme ecosystem. Nous avons trouvé des traces des termes microcosm, proposé par Forbes en 1887, puis community, biotic community, superorganism présents chez Clements dès 1916, puis holocen, utilisé par Friedrichs en 1927, et enfin quasi-organism et ecosystem chez Tansley en 1935.

II y donc un mouvement terminologique qui accompagne la constitution du concept. Lorsqu'une dénomination est définitivement adoptée, elle vient remplacer toute une série de vocables existant déjà et constituant ce que l'on peut appeler un foisonnement terminologique. Bien sûr, on peut s'interroger sur les raisons qui ont favorisé l'adoption du terme ecosystem au détriment des autres. II est toujours difficile de déterminer pourquoi une dénomination en particulier vient finalement remplacer toutes les autres, pour devenir l'expression linguistique stable d'un concept. Peut-être la forme du terme rentre-t-elle en jeu, sa graphie, sa prononciation, son caractère familier, sa transparence aussi ${ }^{11}$, peut-être faut-il également tenir compte de la notoriété du scientifique qui a inventé ou utilisé le terme pour la première fois.

Nous soulignerons simplement que le terme ecosystem mettra un certain temps pour s'implanter dans la communauté scientifique de langue anglaise d'abord, avant d'être traduit dans d'autres langues. D'ailleurs, le manque relatif de popularité du terme au début de son existence amènera d'autres auteurs à proposer des termes concurrents, disparus de la littérature écologique depuis. C'est ainsi qu'en 1939, Thienemann propose d'associer les concepts BIOCOENOSIS à celui de BIOTYPE, pour former une unité supérieure qui correspond au concept BIOSYSTEM , concept homologue d'ECOSYSTEM. Le terme biosystem (qui ne sera, à notre connaissance, jamais traduit en français) sera rarement repris dans la langue anglaise et disparaîtra rapidement au profit d'ecosystem qui s'implante ainsi définitivement.

\subsection{Le cas du terme complexe organisme-milieu}

Nous l'avons déjà souligné, les concepts ECOSYSTEM et ÉCOSYSTÈM E sont apparus à la faveur de travaux conduits simultanément à la fois en Europe et aux États-Unis. Cependant, la simultanéité de ces travaux ne signifie pas pour autant qu'il y ait eu des connexions entre eux ou que les termes utilisés à l'époque étaient automatiquement connus et traduits dans la langue voisine. Nous avons ainsi rencontré un certain nombre de termes anglais et français qui ne possèdent aucun équivalent dans l'une ou l'autre langue, ce qui laisse penser que les travaux dans lesquels ils apparaissaient n'étaient pas connus ou en tout cas pas repris hors des frontières de leur pays d'origine. Ainsi, nous n'avons jamais trouvé de trace, au cours de nos recherches, de termes français qui pourraient être considérés comme les termes équivalents des dénominations anglaises superorganism et quasi-organism. En revanche, les termes microcosme et holocène, bien qu'extrêmement rares, apparaissent ponctuellement dans des ouvrages scientifiques qui relatent la naissance du concept ECOSYSTEM . À I'inverse, certains termes français, et c'est le cas de complexe organisme-milieu, ne sont jamais apparus dans la littérature écologique anglaise.

Le terme complexe organisme-milieu apparait régulièrement dans les ouvrages d'écologie dès la fin des années 1920 et nous avons pu déceler sa présence jusque dans 
le milieu des années 1930. Un des derniers auteurs à utiliser le terme, Prenant, en propose même, en 1934, une modification graphique (1934: 25) :

Pour rejoindre vraiment l'écologie, on n'a pas le droit d'isoler face à face, l'être vivant et le milieu physique. La formule classique féconde du «complexe organisme-milieu » n'est plus ici tout à fait suffisante. II vaudrait mieux préciser qu'il s'agit du «complexe organismes-milieu », c'est-à-dire envisager des ensembles très vastes.

La proposition de Prenant de modifier le terme complexe organisme-milieu en complexe organismes-milieu ne sera semble-t-il jamais reprise par ses contemporains, puisque nous n'avons trouvé aucun ouvrage ultérieur qui mentionne le terme complexe organismes-milieu. D'ailleurs le terme que Prenant se propose de modifier apparaîtra quelques années encore avant d'être remplacé par écosystème dans les ouvrages d'écologie, et ce, dès le début des années 1940.

\section{LES MODIFICATIONS CONCEPTUELLES D'ÉCOSYSTÈME}

\subsection{La délimitation de la taille et de la durée de vie d'un écosystème}

L'analyse diachronique du concept ECOSYSTEM nous a permis de décrire sa constitution et les mouvements terminologiques qui ont accompagné son évolution. Cependant, une fois le terme ecosystem adopté et implanté dans la langue de l'écologie, le concept a continué d'évoluer sans entraîner de modification terminologique parallèle, comme c'était le cas jusqu'alors.

En effet, l'écosystème s'affine encore grâce aux travaux de l'Américain Lindeman qui, en 1941, donna au concept l'extension qu'il possède encore aujourd'hui. Selon lui, un écosystème est un ensemble formé par des éléments biotiques ou vivants et par des éléments abiotiques ou morts. Cet ensemble est de surcroît soumis à des flux énergétiques et à des relations trophiques, (en rapport avec la nourriture). Autrement dit, chaque niveau $n$ de l'écosystème est traité par Lindeman comme un système thermodynamique échangeant de l'énergie avec son environnement biotique et abiotique. Il est en outre considéré pour la première fois comme un système indépendant et peut-être même autosuffisant. Cette nouvelle information conceptuelle apportée par Lindeman est encore aujourd'hui un élément important de la définition de l'écosystème.

$M$ algré tout, même si le concept se clarifie au cours de son évolution, il existe encore aujourd'hui des zones d'ambiguïtés, surtout lorsqu'il s'agit de définir la taille d'un écosystème. En effet, il semble délicat de mesurer l'étendue géographique exacte d'un tel ensemble, qui peut avoir la taille d'une goutte d'eau ou celle de l'océan Atlantique. À cette occasion, nous remarquerons de nouveau qu'il existe des différences entre les terminologies française et anglaise de l'écosystème. Ainsi, la langue anglaise semble avoir trouvé les moyens linguistiques de décrire un écosystème de grande taille, mais il ne semble pas y avoir de terme pour désigner ceux qui, au contraire, sont de petite taille. Nous avons ainsi fréquemment rencontré le terme ecosphere, qui désigne le plus grand écosystème qui soit, c'est-à-dire la planète, un écosystème où l'élément biotique est appelé biosphere et représente, littéralement, l'enveloppe de vie qui entoure la planète. De la même façon, l'anglais utilise le terme ecotope pour désigner les subdivisions qui apparaissent à l'intérieur d'un écosystème de grande taille, comme c'est le cas pour l'écosphère par exemple. 
En revanche, l'anglais semble avoir plus de difficultés pour désigner la zone de transition qui existe entre deux écosystèmes de grande taille alors qu'il existe un terme pour décrire cette zone de transition lorsqu'ils sont de petite taille. C'est le cas du terme ecotone qui, si nous assimilons les écosystèmes à des cellules, représente alors en quelque sorte la membrane cellulaire de l'écosystème. Nous avons en outre remarqué que le terme ecotone ne s'utilise en anglais que lorsqu'il y a une certaine compétition entre les végétaux qui peuplent la zone de transition, le terme tension, étant presque toujours associé à celui d'ecotone ${ }^{12}$. Puisque cette tension s'observe plus facilement dans les écosystèmes de petite taille que dans ceux qui couvrent un espace géographique important, le terme ecotone tend donc naturellement à décrire des zones de transitions entre les petits écosystèmes.

Le français possède également des moyens terminologiques d'appréhender la taille des écosystèmes et propose des termes équivalents pour désigner les écosystèmes de grande taille. Ainsi les termes écosphère, écotope et écotone désignent-ils les mêmes réalités que les termes anglais décrits ci-dessus.

Par contre, le français semble avoir trouvé un moyen de désigner les écosystèmes de petite superficie et de vie courte, ce que ne fait pas I'anglais. Nous avons fréquemment rencontré le terme synusie qui désigne concrètement un sous-ensemble d'écosystèmes, un peuplement dont la durée de vie est provisoire (un arbre mort par exemple). Le terme anglais vraisemblablement équivalent, synusia, n'apparaît que rarement dans la littérature écologique anglaise (nous ne l'avons rencontré qu'une seule fois, chez Brewer [1979]). II représente pourtant, à notre avis, une tentative de dénommer des écosystèmes de petite taille, puisqu'il insiste sur les limites géographiques mais aussi temporelles des communautés qui forment l'écosystème.

Nous pouvons en effet nous interroger sur la durée de vie de tels ensembles: certains sont-ils provisoires, au contraire d'autres?

Les limites spatiales et temporelles de tels ensembles, parce que mal connues, font encore l'objet de recherches en écologie, et nous nous demandons si toute cette réflexion qui se trouve actuellement en gestation n'aboutira pas finalement à la création de nouveaux termes ou à l'adoption de vocables existant déjà dans d'autres langues ou dans d'autres disciplines. II serait sûrement intéressant de vérifier cette hypothèse dans quelques années.

\subsection{Hypothèses pour le futur}

Nous avons déjà rencontré certains termes nouveaux, en français, qui semblent appuyer cette hypothèse, comme c'est le cas pour géosystème mais aussi pour paysage, redéfini pour l'occasion. Ces deux termes n'apparaissent encore que très rarement dans les ouvrages écologiques et uniquement dans les publications faites depuis 1990. Un géosystème représenterait un ensemble d'écosystèmes de grande taille et serait donc le plus grand écoystème avant l'écosphère. Selon Drouin (1991: 43) :

Les écosystèmes s'emboîtent, se chevauchent, se superposent. Leur délimitation spatiale n'est pas seulement problématique, elle est souvent arbitraire. Pour répondre à des questions de ce type, il est nécessaire de disposer d'un niveau d'intégration supérieur qui englobe plusieurs écosystèmes de la même façon que ceux-ci englobent les peuplements eux-mêmes formés de populations. C'est dans cet esprit qu'ont été proposés des 
concepts comme ceux de «géosystèmes » ou de «paysage». Leur statut théorique semble encore en débat, mais leur importance pratique est évidente.

Enfin, nous remarquions au début de cet article que depuis le début des années 1970, il s'était opéré une véritable césure au niveau du concept ÉCOLOGIE, qui représentait maintenant quasiment deux sciences différentes. En effet, il est difficilement possible d'assimiler l'écologie qui, schématiquement, réfléchit sur les rapports entre les végétaux et les animaux entre eux et avec leur milieu de vie, et ce que l'on appelle de plus en plus fréquemment l'écologisme qui dénonce les conséquences de l'industrialisation à l'échelle humaine et qui s'est formé en partis politiques. Nos recherches nous ont montré que le concept ÉCOSYSTÈME tendait à suivre la même évolution que celui d'ÉCOLOGIE, puisque depuis peu l'écosystème est envisagé par les scientifiques comme un ensemble fonctionnant à échelle humaine et pouvant résoudre les problèmes causés par l'industrialisation à outrance. N ous ne doutons pas ici aussi que cette tendance ne se traduise, à terme, par la proposition de nouveaux concepts et de nouveaux termes. Nous avons déjà rencontré le terme agroécosystème ${ }^{13}$ (dont nous n'avons encore pas trouvé d'équivalent en anglais) qui désigne, dans un milieu rural, I'unité écologique d'un système agricole. L'agroécosystème est tourné entièrement vers l'homme dans le monde rural et désigne ses techniques de travail, son alimentation et son organisation. II associe donc l'économie régionale, le régime alimentaire des habitants et des animaux domestiques, les terrains et les techniques agricoles.

Étant donné l'importance que prend l'homme et son milieu dans la définition des concepts clés de l'écologie, nous ne doutons pas que le terme agroécosystème ne soit le premier avènement terminologique de toute une série de termes à venir. Les grands concepts de la science écologique semblent devoir donner naissance à des concepts voisins, ne concernant que les hommes.

\section{CONCLUSION}

À travers l'analyse présentée ici, nous avons tenté de souligner l'importance de la terminologie et sa richesse pour la traduction, surtout si l'on s'attache à réfléchir sur la dimension diachronique et comparative de la terminologie. Un des points essentiels que nous avons voulu mettre en valeur concerne la nécessité pour le traducteur de vivre en bonne intelligence avec le concept qu'il traduit, c'est-à-dire la nécessité qu'il a de connaître le domaine d'origine du concept, les diverses dénominations qui l'ont désigné, l'évolution historique qu'il a connue et sa définition actuelle. Ces préalables à l'acte de traduction peuvent paraitre trop ambitieux, voire excessifs si l'on prend en compte l'état d'urgence dans lequel travaillent toujours les traducteurs. Pourtant, ces préalables permettent indéniablement de mieux connaître le concept et donc de le traduire dans de meilleures conditions, en s'éloignant d'une traduction littérale du signe qui, lorsqu'elle est systématiquement appliquée, peut conduire à des équivalences médiocres. Cette connaissance quasiment «intime» du concept apparaît d'autant plus souhaitable lorsque les traducteurs se trouvent face à des concepts que nous avons appelé «anodins», qui leur semblent connus, à tort. Nous l'avons vu avec les concepts COMMUNAUTÉ et COM MUNITY, l'équivalence notionnelle n'est en effet pas toujours de mise entre deux langues voisines même lorsque les termes apparaissent, eux, comme équivalents. 
Nous souhaitons souligner de nouveau à quel point la terminologie est un outil de travail formidable pour la traduction, surtout lorsque la dimension diachronique, accompagnée de la dimension comparative, vient parfaire l'outil terminographique. Les recherches sur l'archiconcept menées par Thoiron et al.(1994) avaient déjà attiré l'attention des terminologues sur ce point.

Cette réflexion sur la traduction spécialisée menée par le biais de la terminologie nous amène également à considérer qu'il existe deux catégories de traducteurs spécialisés. Il y a d'abord les traducteurs spéciali sés spécialistes, scientifiques à l'origine, et qui bien souvent, sont des écologues devenus traducteurs et terminologues. Il y a également ceux que nous appellerons les traducteurs spécialisés non-spécialistes qui sont traducteurs et terminologues de formation et qui viennent à l'écologie pour les besoins de leur profession.

La différence n'est triviale qu'en apparence. Les besoins de ces deux types de traducteurs sont différents: le traducteur spécialiste maîtrise bien les concepts de son domaine mais peut souhaiter davantage d'informations de nature strictement linguistique, alors que le traducteur non-spécialiste a besoin d'indications fines sur les contenus conceptuels.

Cette réalité demande une réflexion adaptée de la part du terminologue qui crée les outils d'aide à la traduction. Nous pensons que les traducteurs spécialistes et les traducteurs non-spécialistes n'ont pas besoin des mêmes outils terminographiques et peut-être est-ce là une piste de recherche nouvelle en terminologie que de chercher à mettre au point des outils de travail différents pour ces deux catégories de traducteur.

\section{NOTES}

1. Dans cet article, nous laissons de côté le débat qui distingue la notion du concept et, conformément aux recommandations de l'Association Française de Normalisation (AFNOR), nous considérons les deux termes comme équivalents. Nous employons donc ici indifféremment les termes notion et concept.

2. L'article utilise les codes typographiques suivants: les concepts en langue française sont en caractères majuscules, gras et italiques (CONCEPT), alors que les concepts en langue anglaise sont en caractères majuscules italiques (CONCEPT). Les termes français sont typographiés en caractères minuscules, gras et italiques (terme), les termes en langue anglaises se trouvent en caractères minuscules, italiques (terme).

3. Le terme 0 ekologie a été utilisé pour la première fois par le zoologiste allemand Ernst $\mathrm{H}$ aeckel en 1866, mais le véritable essor de l'écologie en tant que science date réellement du début du XX ${ }^{\circledR}$ siècle.

4. C'est le cas notamment de Duvigneaud, dans son ouvrage de 1974.

5. Le concept BIOSPHÈRE a été emprunté au domaine de la biogéographie, qui elle-même le tient de la biologie, où la biosphère représente un atome qui serait à l'origine des corps organisés mais dont l'existence est hypothétique. Pris dans un sens écologique, la biosphère représente, schématique ment, la région de la planète qui renferme l'ensemble des êtres vivants et dans laquelle la vie est possible en permanence. Le concept CLIM AX est une ancienne figure rhétorique grecque indiquant la gradation. Le terme, devenu archaïque, a été utilisé pour la première fois dans un sens écologique en 1916 par Clements qui décrit le climax comme le stade ultime et quasiment idéal de développement d'une communauté végétale. Le concept NICHE désignait à l'origine un emplacement creux, un enfoncement pratiqué dans un mur pour y loger une statuette. Après être tombé en désuétude, le terme a subi une transgression sémantique et a été introduit en écologie par Grinnel, en 1916, pour désigner l'espace géographique et la position qu'occupe une espèce vivante à l'intérieur d'une communauté.

6. Un schéma retraçant l'évolution diachronique des concepts ÉCOSYSTÈME et ECOSYSTEM, ainsi qu'une fiche terminologique bilingue sont proposés en appendices en fin d'article.

7. Peut-être faudrait-il utiliser ici le conditionnel, car bien que la plupart des ouvrages historiques reconnaissent en Tansley le créateur du terme ecosystem, il se peut néanmoins que des recherches 
ultérieures sur le terme montrent qu'il avait déjà été utilisé avant 1935 par un autre auteur. Nous n'avons pu, dans nos recherches, trouver la trace du terme avant 1935 et nous considérons donc pour l'instant que, selon toute vraisemblance, Tansley est effectivement celui qui l'a utilisé pour la première fois, mais il convient toujours de rester prudent lorsqu'il s'agit d'attribuer la naissance d'un terme à un scientifique.

8. Notre traduction.

9. Tansley écrit (1935: 199) : «I plead for empirical method and terminology in all work on vegetation, and avoidance of generalised interpretation based on a theory of what must happen because « vegetation is an organism». [...] I have criticised the term «organism» as applied to communities of plants or animals, or to «communities» of plants and animals, on the ground that while these aggregations have some of the qualities of organisms (in the biological sense), they are too different from these to receivethe same unqualified appellation. »

10. Whittaker propose la définition suivante de community (1975:1): «The community is a functional system of interacting populations but this definition ignores the questions of how this system of species is organized.»

De même, on trouve la définition suivante chez Brewer (1979: 284) : «Community : a group of organisms occupying a particular area, the connotation is of a coacting system.»

11. C'est ce que montrent Thoiron et al. (1994) dans une enquête sur l'implantation des termes de santé et de médecine.

12. Clarke note (1954: 43) : «The transition zone of tension between communities presents a situation of special interest and is known as an ecotone. [...] The transition zone sometimes more than $100 \mathrm{~km}$ in width between major continental communities are regarded by some as ecotones, but others restrict the term to areas of smaller scale. Strictly speaking a transition area is an ecotone only if tension exists between the bordering communities, and this is often difficult to demonstrate, especially for large areas. 》

13. Chez Duvigneaud (1974: 202) : «L'agroécosystème est un ensemble d'agrobiocénoses reliées écologiquement entre elles par des facteurs climatiques, édaphiques, topographiques, biotiques, mais dépendant aussi de l'économie régionale, qui comprend les traditions et techniques agricoles et le régime alimentaire des habitants».

\section{RÉFÉRENCES}

Agu esse, P. (1971) : Clefs pour l'écologie, Paris, Seghers, 219 p.

Br ewer, R. (1979) : Principles of Ecology, Philadel phia, Saunders College, 299 p.

Cand ol l e, A. de (1855) : Géographie botanique raisonnée, Paris, V. M asson, 2 volumes.

Cl ar ke, G. L. (1954) : Elements of Ecology, New York, John Wiley and Sons, London, Chapman and Hall, $534 \mathrm{p}$.

Cl ement s, F. E. (1916) : Plant Succession. An Analysis of the Development of Vegetation, Washington, Carnegie Institution, $321 \mathrm{p}$.

D el éage, J. P. (1992) : Histoire de l'écologie. Une science de l'homme et de la nature, Paris, Éditions La Découverte, $330 \mathrm{p}$.

D r ouin, J. M. (1991) : L'écologie et son histoire, Paris, Flammarion, 213 p.

Dury, P. (1997): Étude comparative et diachronique de l'évolution de dix dénominations fondamentales du domaine de l'écologie en anglais et en français, Thèse de Doctorat Nouveau Régime, sous la direction de Philippe Thoiron, Université Lumière Lyon II, 425 p.

Duvigneaud, P. (1974) : La synthèse écologique. Populations, écosystèmes, biosphère, noosphère, Paris, Douin Éditeurs, $291 \mathrm{p}$.

Egert on, F. N. (1977) : History of American Ecology, New York, Arno Press, 370 p.

Grinnel, J. (1917): «The Niche-Relationships of the California Trasher», The Auk, A Quaterly Journal of Ornithology, 34-4, pp. 427-433.

Lin deman, R. L. (1941) : «Seasonnal Food-Cycle Dynamics in a Senescent Lake», Am. M id. Nat., 26, pp. 636-673.

Prenant, M . (1934) : Adaptation, écologie et biocénotique, Paris, Hermann \& Cie, 60 p.

Tansl ey, A. G. (1935) : «TheUse and Abuse of Vegetational Concepts and Terms», Ecology, 16-3, p. 299. 
Thien emann, A. (1939) : «Grundzüge einer allgemeinen Ökologie», Arch. Hydrobiolo., 35, pp. 267285.

Th oir on, P. (1994) : «Terminologie multilingue: une aide à la maîtrise des concepts», M eta, 344, pp. 765-773.

- - (1995) : Concepts, termes et traduction, Communication présentée lors du $120^{e}$ congrès national des sociétés historiques et scientifiques, Aix-en-Provence, octobre 1995, pp. 1-8.

Thoir on, P. et al. (1994) : «Résultats de l'enquête d'implantation des termes de santé et de médecine», Terminologies nouvelles, 12, pp. 77-88.

War ming, E. (1909, rééd. de 1895) : O ecology of Plants, traduit de Lehrbuch der ökologischen Pflanzengeographie, Oxford, Oxford University Press, $166 \mathrm{p}$.

Wh it taker, R. H. (1975) : Communities and Ecosystems, New York, Macmillan, 385 p.

\section{APPENDICE I}

Diagramme synthétique retraçant l'évolution des concepts ECOSYSTEM/ÉCOSYSTÈME

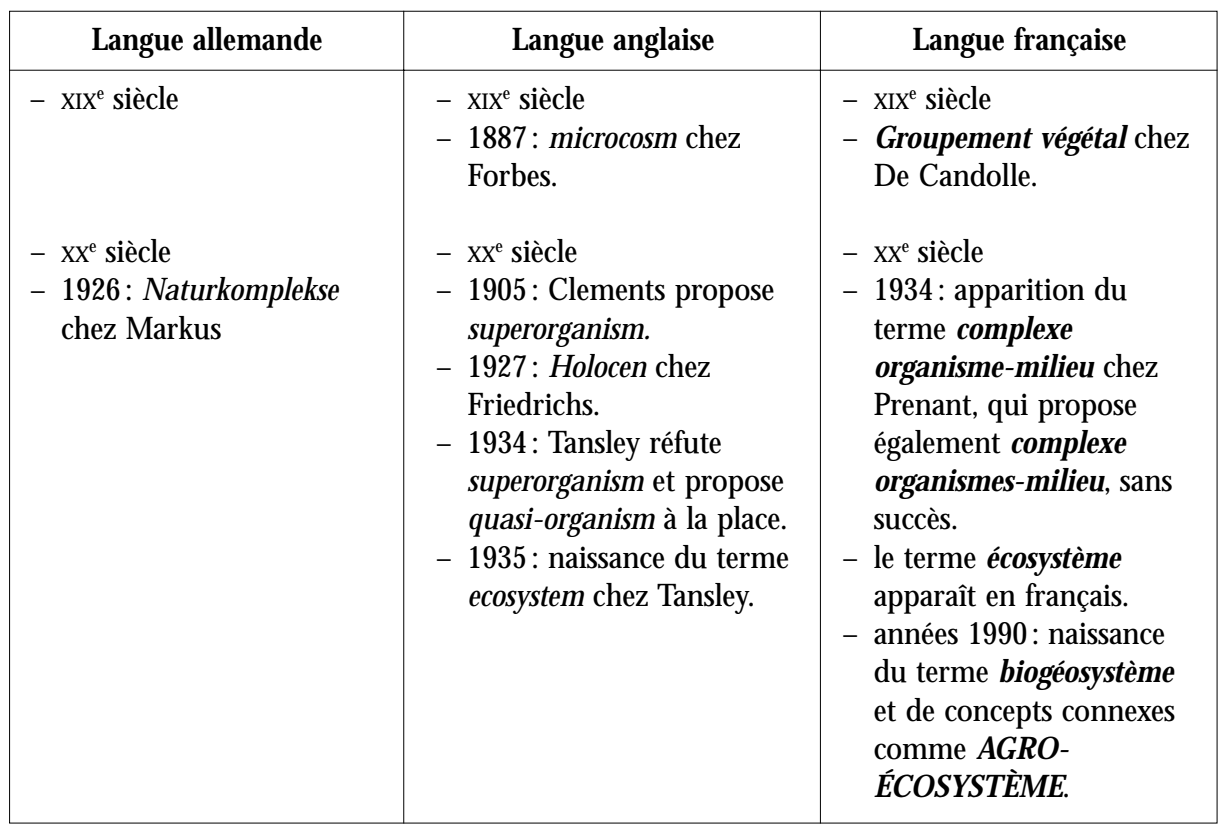

\section{APPENDICE II}

\section{Fiche terminologique bilingue}

Vedette anglaise: ecosystem

nombre: $\mathrm{s} \quad$ catégorie: $\mathrm{n}$

synonyme (s) :

renvois: biosphere, biotic community, biome.

étymologie: grec [oïkos], maison et [sustêma], système.

corrélats terminologiques: ecosphere, biotic community, abiotic community, ecotope, ecotone, biosphere.

\section{Définition de la vedette anglaise:}

(1935/TAUAV.299). Our natural human prejudices force us to consider the organisms [...] as the most important parts of these systems, but certainly the inorganic «factors» are also parts there could be no systems without them, and there is constant interchange of the most 
various kinds within each system, not only between the organisms but between the organic and the inorganic. These ecosystems, as we may call them, are of the most various kinds and sizes.

(1990/BHTE.851). Ecosystem: a holist concept of the plants, the animals habitually associated with them, and all physical and chemical components of the immediate environment, or habitat which together form a recognizable self-contained entity.

Contexte (s) :

(1986/ON HCE.5). Unless recognizable boundaries exist, there is no simple method for specifying the size of an ecosystem.

\section{Évolution diachronique:}

- $\quad$ 1935: création du terme par l'anglais Tansley pour désigner un concept qui existe depuis longtemps déjà et qui est apparu auparavant sous d'autres formes qui ne resteront pas dans la langue: microcosm (Forbes, 1887), Naturkomplekse (Markus, 1926), holocen (Friedrichs, 1927), quasi organism (Tansley, 1930).

- 1936: Clements, aux États-Unis, développe la vision dynamique des écosystèmes, en développant le concept de biotic community.

- 1939: Parallèlement à Tansley, l'Américain Thienemann invente le terme biosystem avec le même sens que ecosystem, mais biosystem ne sera pas repris dans la littérature écologique.

- $\quad 1941$ : l'Américain Lindeman affine le concept ec osyst em : ce dernier devient un ensemble d'êtres vivants et morts soumis à des flux énergétiques.

- $\quad$ II subsiste encore une ambiguïté notionnelle quant à la taille d'un écosystème. De nouvelles dénominations apparaissent pour lever cette ambiguïté: ecosphere (le plus grand écosystème qui existe), ecotope (les subdivisions qui s'opèrent à l'intérieur d'un écosystème de grande taille), ecotone (la zone de transition entre deux écosytèmes).

Vedette française: écosystème

nombre: $s$

synonyme (s) :

renvois: biocénose, biosphère.

étymologie: idem

corrélats terminologiques: biospère, écotone, écotope, biotope, biocénose, synusie, microécosystème, macroécosystème, mésoécosystéme, agroécosystème.

\section{Définition de la vedette française:}

(1934/PRAEB.25). La formule classique féconde du «complexe organisme-milieu » n'est plus ici tout à fait suffisante. II vaudrait mieux préciser qu'il s'agit du «complexe organismes-milieu », c'est-à-dire envisager des ensembles très vastes.

(1978/LBECT.1). Un écosystème comporte un ensemble d'éléments vivants, végétaux et animaux, qui constituent une biocénose et le milieu physique au sein duquel vivent les êtres vivants, milieu parfois qualifié de biotope.

(1979/DUPAE.31). Les écosystèmes sont des systèmes relativement homogènes où un équilibre particulier s'établit dans des réseaux alimentaires bien spécifiques.

(1986/DAPRE.262). La biocénose et son biotope sont deux éléments indissolublement liés qui réagissent l'un sur l'autre pour former un système plus ou moins stable qui a reçu le nom d'écosystème. 


\section{Contexte (s) :}

(1991/DRECH/106). Les écosystèmes s'emboîtent, se chevauchent, se superposent. Leur délimitation spatiale n'est pas seulement problématique, elle est souvent arbitraire. [...] C'est dans cet esprit qu'ont été proposés des concepts comme ceux de «géosystème» ou de «paysage».

(1995/COIDE/47). Le problème est que pareilles usines sont fortement convoitées, de sorte que les «micro-écosystèmes» que constituent les mollusques sont des lieux privilégiés pour la compétition entre Trématodes d'espèces différentes.

\section{Évolution diachronique:}

- Années 1920: le français utilise le terme complexe organisme-milieu, précurseur du terme écosystème. Prenant, en 1934, préconise l'emploi de complexe organismes-milieu, sans succès. Le terme complexe organisme-milieu a été remplacé par écosystème et a disparu dela littérature écologique.

- Tout comme dans la langue anglaise, on retrouve les mêmes difficultés à décrire linguistiquement les limites spatiales d'un écosystème. En français, une synusie décrit un écosystème de petite taille, dont le peuplement a une durée de vie limitée (un arbre mort par exemple).

- $\quad$ On trouve également depuis peu les termes macro-, méso-, et microécosystème.

- Les ouvrages contemporains mentionnent parfois le terme biogéosystème souvent considéré comme un synonyme de paysage, et qui désigne un écosystème de très grande taille. II ne semble pas exister d'équivalent en anglais.

- Le concept écosystème semble avoir donné naissance au concept agroécosystème, qui ne concerne que l'homme dans son milieu rural. 